\title{
ADAPTAÇÃO DA REGRA DE TAYLOR À REALIDADE BRASILEIRA PARA DETERMINAÇÃO INICIAL DA TAXA DE PRODUÇÃO EM PROJETOS MINEIROS
}

Jorge Luiz Valença Mariz' Rodrigo de Lemos Peroni ${ }^{1}$

\section{Resumo}

Um projeto de mineração geralmente envolve três estágios de avaliação, que são os estudos conceitual, de pré-viabilidade e de viabilidade, que necessitam da definição de um calendário de produção e da expectativa de vida útil do empreendimento, mesmo quando as informações acerca dos recursos são ainda conjecturas. Existem alternativas para estimar estes dados através de modelos empíricos, embora tenha sido verificado que estas não apresentam aderência à realidade brasileira quando confrontados com dados obtidos junto à Agência Nacional de Mineração entre os anos 2010 a 2015. Através de Simulações de Monte Carlo, foi possível desenvolver equações para determinação da taxa de produção inicial com maior aderência a cada cenário proposto. A primeira análise considerou a segmentação dos dados por substância mineral, ao passo que a segunda considerou simultaneamente a segmentação dos dados por substância mineral, método de lavra e porte do empreendimento. Conclui-se que os métodos clássicos de estimativa de taxa de produção e vida útil não são aplicáveis aos empreendimentos mineiros em operação no Brasil, ao passo que as equações propostas neste trabalho oferecem alternativas mais aderentes para cada cenário analisado.

Palavras-chave: Planejamento de lavra; Regra de Taylor; Taxa de produção; Vida útil.

\section{ADAPTATION OF TAYLOR'S RULE TO BRAZILIAN REALITY FOR INITIAL DETERMINATION OF THE PRODUCTION RATE IN MINING PROJECTS}

\begin{abstract}
A mining project generally involves three stages of evaluation, which are the conceptual, pre-feasibility and feasibility studies, which require the definition of a production schedule and the lifetime expectancy of the enterprise, even when information about resources are still conjectures. There are alternatives to estimate this data through empirical models, although it was verified that these are not adhering to the Brazilian reality when faced with data obtained from the National Mining Agency between the years 2010 to 2015. Through Monte Carlo simulations, it was possible develop equations to determine the initial production rate with greater adherence to each proposed scenario. The first analysis considered the segmentation of the data by mineral substance, while the second considered simultaneously the segmentation of the data by mineral substance, mining method and size of the enterprise. It was concluded that the classical methods of estimation of production rate and lifetime are not applicable to mining projects in operation in Brazil, whereas the equations proposed in this work offer more adherent alternatives for each scenario analyzed.
\end{abstract}

Keywords: Mine planning; Taylor's rule; Production rate; Lifetime.

\section{INTRODUÇÃO}

A obtenção de valores coerentes para a taxa de produção de uma mina e sua vida útil não é uma questão trivial, visto que taxas de produção reduzidas alongam o fluxo de caixa, postergam receitas e sacrificam potenciais lucros. Por outro lado, taxas de produção elevadas aumentam consideravelmente os custos de capital, levando o empreendimento a sequer recuperar o investimento por conta da vida útil reduzida, quando eventualmente produziriam mais do que a capacidade de absorção do mercado. A busca por respostas para essa questão teve início em 1977, quando Taylor analisou 30 minas em operação ou projetos aptos ao início, cujos tamanhos e geometrias dos corpos minerais eram diversos (excluindo

'Departamento de Engenharia de Minas, Universidade Federal do Rio Grande do Sul - UFRGS, Porto Alegre, RS, Brasil. E-mail: jorge_valenca@ hotmail.com

2176-1523 (C) 2019 Associação Brasileira de Metalurgia, Materiais e Mineração. Publicado pela ABM. Este é um artigo de acesso aberto distribuído sob os termos da licença Creative Commons CC BY-NC-ND (Attribution-NonCommercial-NoDerivs) - https:// creativecommons.org/licenses/by-nc-nd/4.0/ 
depósitos tabulares). $\mathrm{O}$ autor considerou depósitos com reservas razoavelmente conhecidas e averiguou que a taxa de extração comportava-se proporcionalmente a três quartos da massa do depósito, onde a vida útil seria proporcional à raiz quádrupla da massa total. $O$ estudo propôs uma equação da forma $\mathrm{y}(\mathrm{x})=\mathrm{a} * \mathrm{x}$, conforme a equação $\mathrm{I}$ :

$$
\boldsymbol{L} \cong 0,2 * \boldsymbol{T}^{0,25}
$$

onde $\boldsymbol{L}$ representa a vida útil da mina, em anos, e $\boldsymbol{T}$ representa a massa de minério do depósito. É indiferente o uso da tonelada métrica ou curta, graças à atenuação da divergência pela raiz quádrupla (equivalente a elevar o $T$ a 0,25) [I-4].

Camm desenvolveu, em 1991, um estudo que buscava obter modelos de custos para aplicar na avaliação de pré-viabilidade de empreendimentos, onde o autor chegou à conclusão de que a capacidade produtiva por dia podia ser calculada como na equação 2 :

$$
C_{s t}=\frac{T_{s t}}{D w y * L}
$$

onde $C_{\boldsymbol{s} t}$ representa a capacidade produtiva por dia em toneladas curtas, e $T_{s t}$ representa a massa de minério existente no depósito em toneladas curtas, $D w y$ representa dias trabalhados em um ano e $\boldsymbol{L}$ representa a vida útil do depósito. A vida útil foi obtida através da aplicação da regra de Taylor, possibilitando ao autor a conclusão de que a relação entre taxa de produção e massa de minério para 350 dias de trabalho por ano correspondia à equação 3 :

$$
\boldsymbol{C}_{\boldsymbol{s t}}=0,0143 * \boldsymbol{T}_{\boldsymbol{s t}}{ }^{0,75}
$$

onde $\boldsymbol{C}_{\boldsymbol{s} t}$ representa a capacidade produtiva por dia em toneladas curtas, e $T_{s t}$ representa a massa de minério existente no depósito em toneladas curtas [5]. Desde então, a equação sofreu diversas revisões, como as efetuadas por Singer, Menzie e Long [6,7], Long e Singer [8] e Long [9]. A Tabela I apresenta as equações obtidas desde então, todas convertidas para o Sistema Internacional de Unidades (S.I.), onde $\boldsymbol{C}$ representa a taxa de produção em toneladas e $\boldsymbol{T}$ representa a reserva mineral, também em toneladas.

Mariz e Peroni [3] verificaram que estas metodologias não apresentavam aderência aos empreendimentos operando no Brasil entre 2010 e 2015, conforme os Relatórios Anuais de Lavra (RALs) obtidos junto à Agência Nacional de Mineração
(ANM). A aderência foi verificada evento por evento de forma binária, onde a inserção da taxa de produção declarada nas equações presentes na Tabela I poderia resultar em uma reserva coincidente com a declarada naquele evento (caso positivo) ou não (caso negativo). Foi considerada ainda a tolerância de $20 \%$ para mais ou para menos no enquadramento desta reserva. $O$ banco de dados analisado possui 53.524 processos codificados, que resultaram em 321 . 138 dados de produção anual e reservas que, após a exclusão dos dados espúrios, resultaram em 7.946 eventos. Conforme Long [9], uma mesma mina poderia representar vários eventos caso cada ano analisado se enquadrasse nas restrições propostas. A partir dessas análises, foi identificada a necessidade de desenvolver equações que oferecessem soluções alternativas ao problema e que fossem adaptados às características da mineração brasileira. Mariz e Peroni [3] propuseram uma segmentação de dados por substância mineral, conforme a Tabela 2, que apresenta os 30 minerais mais significativos presentes nos RALs brasileiros, seja pelas maiores produções anuais ou pelos maiores recursos minerais. Foi proposta ainda a adição de um novo coeficiente ao formato proposto por Taylor [4], visto que esta solução permitiu incrementar a aderência das equações aos dados sem que o fosse perdida a simplicidade do modelo, adquirindo a forma $y(x)=a * x^{b}+c$.

Embora as novas equações apresentassem aderências superiores às até então desenvolvidas, foram cogitadas outras segmentações que produzissem equações para cenários mais restritos, tentando assim minimizar a dispersão dos subdomínios. Como resultado, foi proposto um conjunto de equações obtido através da segmentação simultânea por substância mineral, método de lavra e porte do empreendimento efetuada no mesmo banco de dados do estudo anterior.

\section{METODOLOGIA}

O método de Monte Carlo é uma técnica matemática experimental que utiliza variáveis aleatórias em suas soluções, sendo frequentemente aplicado quando a resolução de um determinado problema está além dos recursos disponíveis na matemática teórica. Em casos probabilísticos, a abordagem de Monte Carlo utiliza números aleatórios para simular os

Tabela I. Descrição dos objetos de estudo e das equações obtidas por cada autor

\begin{tabular}{lllc}
\hline \multicolumn{1}{c}{ REFERÊNCIA } & EQUAÇÃO PROPOSTA & \multicolumn{1}{c}{ TIPOS DE MINAS } & $\mathbf{N}^{\circ}$ MINAS \\
\hline Taylor [4] & $\boldsymbol{C}=0,0158 * \boldsymbol{T}^{0,75}$ & Desconhecido & 30 \\
Singer et al. [6] & $\boldsymbol{C}=0,4584 * \boldsymbol{T}^{0,5874}$ & Céu aberto (ouro e prata) & $4 \mathrm{I}$ \\
Singer et al. [7] & $\boldsymbol{C}=0,0273 * \boldsymbol{T}^{0,704}$ & Subterrânea (grandes sulfetos) & 28 \\
Long e Singer [8] & $\boldsymbol{C}=0,026 * \boldsymbol{T}^{0,74}$ & Céu aberto (cobre) & 45 \\
Long OP [9] & $\boldsymbol{C}=0,123 * \boldsymbol{T}^{0,649}$ & Céu aberto e block caving & 342 \\
Long UG [9] & $\boldsymbol{C}=0,297 * \boldsymbol{T}^{0,563}$ & Subterrânea (- block caving) & 197 \\
\hline
\end{tabular}


Tabela 2. Coeficientes que resultaram na equação ótima para as 30 principais substâncias minerais estudadas

\begin{tabular}{|c|c|c|c|c|c|}
\hline SUBSTÂNCIA & EVENTOS & ADERÊNCIA & COEF. A & COEF. B & COEF. C \\
\hline Anatásio & 6 & $100,00 \%$ & 4,60648 & 0,49646 & 0,70342 \\
\hline Areia & 639 & $35,84 \%$ & 0,24556 & $0,3826 I$ & 336,30000 \\
\hline Argilas Comuns & 337 & $37,39 \%$ & 0,16420 & 0,30684 & 355,91505 \\
\hline Argilas Refratárias & 99 & $47,47 \%$ & 0,39798 & 0,40346 & 247,56543 \\
\hline Bauxita Metalúrgica & 105 & $32,38 \%$ & 9,35242 & 0,30453 & 204,99734 \\
\hline Brita e Cascalho & 2112 & $22,54 \%$ & 0,83027 & 0,42917 & 297,81170 \\
\hline Calcário & 787 & $21,22 \%$ & 0,40677 & 0,48719 & 73,66004 \\
\hline Carvão Mineral & 75 & $33,33 \%$ & 0,12961 & 0,60025 & 223,91749 \\
\hline Cassiterita (Primário) & 22 & $40,91 \%$ & 0,43222 & 0,54947 & $27,77 \mid 36$ \\
\hline Cassiterita (Secundário) & 29 & $44,83 \%$ & I,04642 & 0,38920 & 134,62646 \\
\hline Caulim & 50 & $44,00 \%$ & 9,42578 & 0,25393 & 160,61393 \\
\hline Cianita e Outros Refratários & 6 & $100,00 \%$ & 5,91708 & 0,45577 & 2,22864 \\
\hline Cobre & 49 & $32,65 \%$ & 0,02217 & 0,74463 & 20,30153 \\
\hline Criolita & 6 & $66,67 \%$ & 2,04157 & 0,47107 & 2,98060 \\
\hline Dolomito & 93 & $30,11 \%$ & 3,04005 & 0,32661 & 105,41135 \\
\hline Ferro & 369 & $20,60 \%$ & 0,02184 & 0,74319 & 22,84216 \\
\hline Filito & 29 & $34,48 \%$ & 0,03983 & 0,70991 & 158,47558 \\
\hline Fosfato & 55 & $25,45 \%$ & $0,04 \mid 45$ & 0,67519 & 342,54380 \\
\hline Ilmenita & 8 & $87,50 \%$ & $0,0468 I$ & 0,71025 & 17,80874 \\
\hline Magnesita & 21 & $80,95 \%$ & 0,75363 & 0,41957 & 16,92052 \\
\hline Manganês & 46 & $39,13 \%$ & 9,76409 & 0,37419 & 148,20936 \\
\hline Níquel & 35 & $48,57 \%$ & 0,04934 & 0,69976 & 41,39949 \\
\hline Ornamental (Granito, Gnaisse e afins) & 272 & $26,84 \%$ & 3,65362 & 0,31524 & 279,57782 \\
\hline Ouro (Primário) & 136 & $39,71 \%$ & 0,03200 & 0,73177 & 95,99154 \\
\hline Paládio & 6 & $100,00 \%$ & 0,08655 & 0,68038 & 0,36570 \\
\hline Pirocloro & 11 & $45,45 \%$ & 9,92018 & 0,37200 & 247,72353 \\
\hline Potássio & 6 & $83,33 \%$ & I,27268 & 0,43052 & $0,4774 I$ \\
\hline Saibro & 91 & $32,97 \%$ & 2,74737 & 0,26449 & 303,93582 \\
\hline Sal-gema & 12 & $100,00 \%$ & 8,56047 & 0,25577 & 0,66417 \\
\hline Zirconita (Primária) & 8 & $75,00 \%$ & 0,17995 & 0,59434 & 0,20201 \\
\hline
\end{tabular}

processos aleatórios do problema original, inferindo a solução desejada através do comportamento destes números. Neste estudo foi utilizado o software Risk Simulator 2017, definindo os intervalos de variação dos parâmetros da simulação e as distribuições estatísticas que restringiriam as possibilidades de resultados de cada coeficiente em cada simulação efetuada. Inicialmente, Mariz e Peroni [3] já haviam identificado que os resultados obtidos através de equações com três coeficientes $(\mathrm{y}(\mathrm{x})=\mathrm{a} * \mathrm{x}+\mathrm{c})$ apresentavam maior aderência que as equações com dois coeficientes. Destarte, foram utilizadas distribuições estatísticas uniformes na obtenção dos valores de cada coeficiente, sendo definidos arbitrariamente os limites para o coeficiente "a" entre 0 e 10 , para o coeficiente "b" entre 0,25 e I e, por fim, os limites para o coeficiente "c" entre 0 e 500 [ I 0,1 I I]. Nesta seleção foram considerados os intervalos utilizados pelos outros autores, havendo ainda uma ampliação visando assegurar que houvesse mais possibilidades de combinações na busca pela aderência máxima a cada cenário.
Esta aderência foi verificada individualmente de forma binária, onde cada evento foi classificado como aderente quando a reserva declarada no evento, ao ser confrontada com os coeficientes propostos por cada simulação, resultasse na taxa de produção dividida por 350 dias (conforme Camm) [5] ou dentro do intervalo de $20 \%$ para mais ou para menos. Caso o resultado da simulação para um determinado evento fosse uma taxa de produção fora deste intervalo de tolerância, o evento era classificado como não aderente. As variáveis simuladas que, juntas, apresentaram maior aderência aos eventos de determinado cenário, foram então eleitas as mais representativas daquele cenário, compondo assim a equação "ótima" para um determinado conjunto de dados e condicionantes.

Os outputs do software apresentam resultados com I5 casas decimais e, portanto, seria necessária uma impraticável quantidade de simulações para que a caracterização exaustiva do domínio fosse atingida e a aderência máxima obtida. Para superar essa questão, foi definido que seriam efetuados conjuntos de 100.000 simulações até não haver 
mais incremento na aderência em comparação com o conjunto anterior, ou esta aderência decrescesse. A cada nova sequência, os limites de cada coeficiente foram restringidos, sendo tomados como referência, no mínimo, os coeficientes obtidos nos seis melhores resultados finais da simulação anterior. Desta forma, em no máximo 600.000 simulações foi possível obter a aderência máxima de qualquer cenário estudado neste trabalho [ 10$]$.

\section{RESULTADOS E DISCUSSÕES}

Foram executadas, então, simulações para as três segmentações simultaneamente. $\mathrm{Na}$ segmentação por método de lavra, houve o agrupamento dos eventos lavrados através de métodos subterrâneos com os lavrados em concomitância através de métodos subterrâneos e a céu aberto, ao passo que os limites quanto ao porte do empreendimento consideraram produções anuais entre 100.000 e 1.000 .000 toneladas como de médio porte e os acima de 1.000 .000 como de grande porte, conforme a classificação preconizada pela ANM. Os resultados obtidos através das simulações efetuadas nas 30 principais substâncias minerais produzidas no Brasil entre 2010 e 2015 estão dispostos nas Tabelas 3 e 4, onde os coeficientes que compõem as equações ótimas são apresentados com 5 casas decimais (caso contrário havia uma diferença nos resultados), além da aderência obtida e o número de eventos utilizado para ajustar e modelar cada cenário [10].

Tabela 3. Segmentação por substância mineral, porte do empreendimento e eventos lavrados a céu aberto para as substâncias minerais mais importantes

\begin{tabular}{|c|c|c|c|c|c|}
\hline SUBSTÂNCIA & EVENTOS & ADERÊNCIA & COEF. A & COEF. B & COEF. C \\
\hline Anatásio G* & 6 & $100,00 \%$ & 4,60648 & 0,49646 & 0,70342 \\
\hline Areia G & 19 & $42,11 \%$ & 5,72730 & 0,37619 & 0,69246 \\
\hline Areia $\mathrm{M}^{*}$ ** & 614 & $36,97 \%$ & 0,20409 & 0,39347 & 337,56549 \\
\hline Argilas Comuns G & 7 & $57,14 \%$ & 2,93504 & $0,4 \mid 238$ & 0,01132 \\
\hline Argilas Comuns M & 276 & $4 I, 30 \%$ & 0,02047 & 0,26265 & 363,69862 \\
\hline Argilas Refratárias G & 1 & $100,00 \%$ & 7,28183 & 0,53445 & 0,05368 \\
\hline Argilas Refratárias M & 97 & $47,42 \%$ & 0,37282 & 0,40834 & 246,44031 \\
\hline Bauxita Metalúrgica G & 26 & $53,85 \%$ & 4,44781 & 0,47090 & 1,31242 \\
\hline Bauxita Metalúrgica $M$ & 76 & $42,11 \%$ & 9,19104 & 0,30819 & 173,78818 \\
\hline Brita G & 170 & $32,94 \%$ & 6,28845 & 0,35672 & 146,69242 \\
\hline Brita $M$ & $194 \mid$ & $24,06 \%$ & 6,17738 & 0,31030 & 234,00138 \\
\hline Calcário G & 152 & $35,53 \%$ & 9,85163 & 0,32295 & 482,10225 \\
\hline Calcário M & 625 & $25,12 \%$ & 0,17313 & 0,48309 & 270,42977 \\
\hline Carvão Mineral G & 7 & $57,14 \%$ & 9,93237 & 0,38745 & 363,47525 \\
\hline Carvão Mineral M & 48 & $37,50 \%$ & 4,27695 & 0,2578 I & 306,32689 \\
\hline Cassiterita (Primária) G & 6 & $66,67 \%$ & 3,36919 & 0,44615 & 0,04623 \\
\hline Cassiterita (Primária) M & 15 & $40,00 \%$ & 2,26244 & 0,35157 & 0,53576 \\
\hline Cassiterita (Secundária) G & 4 & $75,00 \%$ & 2,53962 & 0,34567 & 0,10370 \\
\hline Cassiterita (Secundária) M & 25 & $40,00 \%$ & 9,50892 & 0,26503 & 2,66656 \\
\hline Caulim G & 11 & $72,73 \%$ & 0,65112 & 0,51668 & 45,09469 \\
\hline Caulim M & 29 & $62,07 \%$ & 9,80034 & 0,26840 & 104,50465 \\
\hline Cianita e Outros Minerais Refratários G & 6 & $100,00 \%$ & 5,91708 & 0,45577 & 2,22864 \\
\hline Cobre G & 30 & $46,67 \%$ & 0,05008 & 0,69515 & 41,83150 \\
\hline Cobre M & 8 & $37,50 \%$ & I, I5280 & 0,46439 & 0,63285 \\
\hline Criolita G & 6 & $66,67 \%$ & 2,04157 & $0,47 \mid 07$ & 2,98060 \\
\hline Dolomito G & 5 & $100,00 \%$ & 9,26302 & 0,35865 & $|, 8503|$ \\
\hline Dolomito M & 76 & $30,26 \%$ & 8,64456 & 0,27636 & 250,97282 \\
\hline Ferro G & 232 & $28,45 \%$ & 5,06118 & 0,40755 & 207,72935 \\
\hline Ferro M & 137 & $24,82 \%$ & 0,07161 & 0,47669 & 279,45602 \\
\hline Filito $M$ & 17 & $47,06 \%$ & 2,21538 & 0,27716 & $198,4 \mid 372$ \\
\hline Fosfato G & 25 & $40,00 \%$ & 1,13260 & 0,49942 & 39,98047 \\
\hline Fosfato M & 30 & $40,00 \%$ & 3,01303 & 0,25152 & 472,94907 \\
\hline
\end{tabular}

*Minas de grande porte, com produção anual acima de I.000.000 de toneladas.*** Minas de médio porte, com produção anual entre 10.000 e 1.000 .000 de toneladas. 
Tabela 3. Continuação...

\begin{tabular}{|c|c|c|c|c|c|}
\hline SUBSTÂNCIA & EVENTOS & ADERÊNCIA & COEF. A & COEF. B & COEF. C \\
\hline Ilmenita G & 6 & $100,00 \%$ & 9,60346 & 0,42921 & 0,22641 \\
\hline Ilmenita M & 2 & $100,00 \%$ & 1,32519 & 0,43065 & 0,07390 \\
\hline Magnesita G & 6 & $100,00 \%$ & 3,28460 & 0,34369 & 0,22676 \\
\hline Magnesita M & 15 & $80,00 \%$ & 0,17466 & 0,36324 & 320,68245 \\
\hline Manganês G & 16 & $87,50 \%$ & 6,57898 & 0,39513 & 10,96477 \\
\hline Manganês $M$ & 20 & $45,00 \%$ & 5,73152 & 0,30310 & 0,25296 \\
\hline Níquel G & 23 & $56,52 \%$ & 0,05894 & 0,68933 & 62,14327 \\
\hline Níquel M & 5 & $60,00 \%$ & 0,69769 & 0,47992 & I,6427I \\
\hline Ornamental G (Granito, Gnaisse e afins) & 15 & $46,67 \%$ & 7,05156 & 0,35612 & 0,19886 \\
\hline Ornamental M (Granito, Gnaisse e afins) & 251 & $28,69 \%$ & 9,19556 & 0,26118 & 407,29648 \\
\hline Ouro (Primário) G & 45 & $42,22 \%$ & 0,01685 & 0,75727 & 220,80868 \\
\hline Ouro (Primário) M & 34 & $47,06 \%$ & 0,35743 & 0,55855 & 17,10187 \\
\hline Pirocloro G & 7 & $42,86 \%$ & 2,32657 & $0,4346 I$ & 0,88999 \\
\hline Pirocloro M & 4 & $50,00 \%$ & 9,22588 & 0,37877 & 0,09200 \\
\hline Saibro G & 6 & $66,67 \%$ & 4,27661 & 0,37707 & 0,20989 \\
\hline Saibro M & 83 & $36,14 \%$ & 6,11470 & 0,25075 & 305,85360 \\
\hline Zirconita (Primária) G & 6 & $66,67 \%$ & 4,25624 & 0,43432 & 5,43045 \\
\hline Zirconita (Primária) M & 2 & $100,00 \%$ & 2,18676 & 0,39286 & 0,07800 \\
\hline
\end{tabular}

*Minas de grande porte, com produção anual acima de I.000.000 de toneladas.** Minas de médio porte, com produção anual entre 10.000 e I.000.000 de toneladas.

Tabela 4. Segmentação por substância mineral, porte do empreendimento e eventos lavrados através de métodos subterrâneos somados aos eventos lavrados em concomitância através de métodos a céu aberto e subterrâneos para as substâncias minerais mais importantes

\begin{tabular}{lccccc}
\hline \multicolumn{1}{c}{ SUBSTÂNCIA } & EVENTOS & ADERÊNCIA & COEF. A & COEF. B & COEF. C \\
\hline Argilas Comuns G & 3 & $100,00 \%$ & 8,74188 & 0,41386 & 0,21167 \\
Argilas Comuns M & 1 & $100,00 \%$ & 8,48496 & 0,38773 & 0,08247 \\
Calcário M & 1 & $100,00 \%$ & 8,25902 & 0,37643 & $0,424 I 2$ \\
Carvão Mineral G & 2 & $100,00 \%$ & 3,2483 I & 0,4168 & 1,42097 \\
Carvão Mineral M & 7 & $100,00 \%$ & 0,95319 & 0,47985 & 0,26318 \\
Cobre G & 2 & $50,00 \%$ & 4,32446 & 0,40316 & 0,16506 \\
Cobre M & 9 & $88,89 \%$ & 8,14062 & 0,32398 & 1,47556 \\
Manganês G & 3 & $100,00 \%$ & 4,31043 & 0,42573 & 0,23997 \\
Manganês M & 1 & $100,00 \%$ & 6,18582 & 0,35135 & 0,12757 \\
Níquel M & 7 & $71,43 \%$ & 0,28280 & 0,57649 & 5,61742 \\
Ouro (Primário) G & 6 & $100,00 \%$ & 2,99459 & 0,44065 & $0,8853 \mid$ \\
Ouro (Primário) M & 25 & $40,00 \%$ & 5,88552 & 0,32337 & 0,84323 \\
Potássio G & 6 & $83,33 \%$ & 1,27268 & 0,43052 & $0,4774 \mid$ \\
Sal-gema M & 12 & $100,00 \%$ & 8,56047 & 0,25577 & $0,664 I 7$ \\
\hline
\end{tabular}

\section{CONCLUSÕES}

Mariz e Peroni identificaram que as equações existentes para a obtenção da taxa de produção e da vida útil de projetos mineiros incipientes (Taylor, Long, dentre outros) não apresentaram aderência ao cenário brasileiro, o que motivou a busca por equações com maior aderência através da segmentação dos dados por substância mineral. Visando outro incremento nos resultados, foi proposta a segmentação conjunta dos dados por substância mineral, método de lavra e porte dos empreendimentos. Estas novas equações apresentaram maior aderência para todas as substâncias minerais quando comparadas com os métodos presentes na literatura, tendo ainda resultados levemente superiores aos obtidos por Mariz e Peroni, onde a aderência mínima obtida nos evento a céu aberto foi da ordem de $24 \%$ e a mínima obtida pelas minas subterrâneas adicionadas às operadas através de métodos subterrâneos e a céu aberto foi de $40 \%$, sendo que a aderência máxima para ambos os casos chegou em determinados cenários a $100 \%$. Embora a dispersão dos dados impeça resultados superiores em alguns cenários, estes apresentam aderência significativamente maior à realidade brasileira que os trabalhos propostos até então 
por outros autores. A opção de utilizar as 64 equações segmentadas por substância mineral, método de lavra e porte do empreendimento em detrimento das 30 segmentadas somente por substância mineral, embora apresentem aderência levemente superior, deve ser feita somente quando houver fortes indícios do porte ou do método de lavra do futuro empreendimento.

\section{Agradecimentos}

Os autores agradecem à CAPES, à ANM, aos Engenheiros de Minas Camila Lamonato Centeno, Marcos Antônio de Holanda Tavares e Gustavo Alexandre Silva, assim como Antônio Alves Amorim Neto e Adhelbar de Albuquerque Queiroz Filho.

\section{REFERÊNCIAS}

I Lee TD. Planning and mine feasibility study - An owners perspective. In: Proceedings of the 1984 NWMA Short Course 'Mine Feasibility - Concept to Completion' by G.E. McKelvey, compiler. Spokane, WA. Northwest Mining Association 1984.

2 Hustrulid W, Kuchta M. Open pit mining planning \& design. 2. ed. Florida: CRC Press; 2006.

3 Mariz JLV, Peroni RL. Análise da aderência à realidade brasileira dos métodos de previsão da taxa de produção em fases prematuras de projeto. In: Instituto Brasileiro de Mineração. Anais do $9^{\circ}$ Congresso Brasileiro de Minas a Céu Aberto e Minas Subterrâneas; 2018 Agosto 0I-03; Belo Horizonte, Brasil. Brasília, DF: IBRAM; 2018.

4 Taylor HK. Mine valuation and feasibility studies. In: Hoskins JR, Green WR, editors. Mineral industry costs. 2nd ed. Spokane: Northwest Mining Association; 1977. p. I- 17.

5 Camm TW. Simplified cost models for prefeasibility mineral evaluations. Washington: U.S. Bureau of Mines; 1991. 35 p. (Information Circular; 9298).

6 Singer DA, Menzie WD, Long KR. A simplified economic filter for open-pit gold-silver mining in the United States. Menlo Park: U. S. Geological Survey; 1998. 10 p. (Open-File Report; 98-207).

7 Singer DA, Menzie WD, Long KR. A simplified economic filter for underground mining of massive sulfide deposits. Menlo Park: U. S. Geological Survey; 2000. (Open-File Report; 00-349).

8 Long KR, Singer DA. A Simplified Economic Filter for Open-Pit Mining and Heap-Leach Recovery of Copper in the United States. U. S. Geological Survey; 200I. Open File Report 0I-218

9 Long, KR. A test and re-estimation of Taylor's empirical capacity-reserve relationship. Natural Resources Research. 2009; I8(I):57-63. http://dx.doi.org/I0.1007/s I 1053-009-9088-y.

10 Mariz JLV, Peroni RL. Estimativa de taxa de produção em fases prematuras de projeto através de dados segmentados por substância mineral, método de lavra e porte dos empreendimentos. In: Associação Brasileira de Metalurgia, Materiais e Mineração. Anais da $4^{a}$ ABMWeek; 2018 Outubro 02-06; São Paulo, Brasil. São Paulo: ABM; 2018.

II Hammersley JM, Handscomb DC. Monte Carlo methods. London: Chapman And Hall; 1964

Recebido em: 30 Nov. 2018

Aceito em: 15 Maio 2019 